Near finalised version of paper published in Population, Space and Place (2014 online) DOI: $10.1002 / \mathrm{psp} .1858$

\title{
Migration decision making as complex choice. Eliciting decision weights under conditions of imperfect and complex information through experimental methods
}

\author{
Vladimir Baláž \\ Institute for Forecasting \\ Slovak Academy of Sciences \\ Bratislava \\ Slovakia \\ vbalaz@yahoo.com \\ Allan M Williams \\ Surrey Tourism Research Centre \\ Faculty of Business Economics and Law \\ University of Surrey \\ Guildford \\ GU2 7XH \\ allan.williams@surrey.ac.uk \\ Elena Fifeková \\ the Faculty of National Economy of the University of Economics in Bratislava \\ Dolnozemská cesta 1 \\ 85235 Bratislava. Slovak Republic \\ fifekova@gmail.com
}

Acknowledgement: The Leverhulme Foundation and the Centrum Strategických Analýz CESTA Grant no. III/2/2011 


\begin{abstract}
Migration decisions are complex, involving both economic and non-economic considerations, and are often made in conditions that depart significantly from the idealised information assumptions of many models. This paper uses a three-stage experimental research design to analyse migrant decision making in the face of complexity and varying information conditions (complete, imperfect, and overloading). It pays particular attention to differences based on previous migration experiences. It focuses on four main issues: (a) the balance between monetary and non-monetary factors; (b) the computation, via a range of methods, of relative decision weights attached to different factors; (c) the impact of country image in relation to information; and (d) the role of preferences in dealing with missing information. The research examines the decision weights for eight attributes of potential destination countries for a sample of 157 young, educated individuals in Slovakia. The relative advantages and challenges of utilising experimental methods in migration research are illustrated.
\end{abstract}




\section{Introduction}

Migration is a complex decision, which may involve multiple and perhaps conflicting goals. Potential migrants collect and assess information on multiple criteria, or attributes of the destination, and these are judged and weighted before a decision is taken. Contrary to the assumptions of traditional neo-classical theories, potential migrants have to cope both with information overload and imperfect information in different contexts. In practice, missing information is often surrogated by country image and/or existing knowledge about particular countries. There are also complexities about the preferences they express in making decisions. These are not invariable, being partially incumbent and partly constructed when making decisions.

Existing migration research provides limited insights into many aspects of complex migration decision making processes. Most research based on neoclassical approaches assumes that migrants base their choice of destination countries on an effective cost/benefit analysis (Borjas, 1989) and is unable to address issues relating to imperfect information, images, the construction of preferences and decision weights. There has been relatively limited quantitative research which systematically analyses the importance of non-economic factors, alongside economic factors, as determinants of decision making; instead, they tend to be treated as extensions of the human capital notion of 'investment' by the migrants (Davies et al, 2001). This lacuna is recognized (Halfacree, 2004) but has mostly been addressed through qualitative research.

In large part these research gaps reflect the limitations of both the dominant research methodologies and data availability, which have constrained research within the neoclassical framework. In contrast, while experimental research methods offer an alternative approach to these issues, there has been only limited application of experimental research methods in migration studies, with very few exceptions (McKenzie and Yang, 2010; McKenzie et al., 2010). Experimental methods allow simulation of decision making and controlling for the influence of specific factors, and are also replicable (Hole, 2012; Samuelson, 2005). This paper aims to make an original contribution to migration research, though the first systematic application of complex choice concepts and methods to migration, being also one of the few applications of experimental methods, to any aspect of migration. It responds to the limiting assumptions of neo-classical approaches about information availability and processing (Stigler, 1961; Stiglitz, 2000), while paying particular attention to the additional complexity involved in addressing multiple non-economic factors, such as crime, health, language difficulty, personal freedom, life satisfaction and climate.

This paper uses innovative experimental research methods to address the following objectives:

1. To identify individuals' information search patterns relating to migration, specifically with respect to:

- The amount of information processed;

- How judgements are formed in relation to dominant attributes under different conditions: binary vs. complex choice, controlling for image, and limited versus unlimited access to information; 
2. To analyse the decision weights attached to particular criteria, or attributes, especially economic versus non-economic (quality of life);

3. To analyse differences between those with and without previous migration experiences in the judgements and decisions made about potential destinations under variable information conditions.

The paper reviews the relevant theoretical literature, focussing on the incorporation of multiple non-economic factors, information conditions, and preferences. It then outlines the research methods, before presenting and discussing findings for 157 young adults in Slovakia across three experimental stages.

\section{Conceptualization}

\section{Labour migration as a complex decision making process and the incorporation of non-economic factors}

Human capital theories consider both the monetary and non-monetary returns and costs of migration (Sjaastad, 1962: 84). Although non-monetary considerations are considered important, they are also difficult to quantify at the individual level. It is assumed they will not affect the aggregate spatial structure of migration flows, if the individual distribution of tastes is random, and competition is perfect with resources moving effortlessly among places. This is a deeply limiting assumption because migration is not situated in a cultural and social vacuum (de Haas, 2010). Therefore, while economic theories can explain why some social groups are more likely than others to migrate, they can not explain differences in the behaviour of individuals with apparently similar economic characteristics (Massey et al, 2003). This individual variation partly stems from the multiplicity of economic and non-economic factors that influence decision making.

Non-monetary costs/benefits are rarely examined in the literature on labour migration. De Jong (1999: 282) writes that 'Economic and job-related motives tend to be dominant; in interpreting such findings there is little recognition that financial rewards are not necessarily ends in themselves, but are means to satisfy other needs and desires'. De Jong's (1999) work is one of the few exceptions that considers a broad range of migration costs/benefits, including non-monetary ones, and that multiple factors rather than single motives usually inform individual decisions. Experimental methods provide a promising approach to studying features of migration decision making which cannot be examined effectively by classical ex-post studies.

This poses the question of which non-economic factors are most important in the knowledge searches of young adult international migrants. The existing literature is relatively thin and, with a few exceptions (Fisher, 2004), is more concerned with decision making than the search for information per se. Push-pull theories do provide a broader perspective on the determinants of migration, although they tend to be dominated by material or environmental considerations (Skeldon, 1997), and characterised by somewhat arbitrary inclusion of variables (de Haas, 2007: 17). They also tend to assume perfect information and ' ... do not allow for assigning relative 
weights to the different factors affecting migration decisions' (de Haas, 2007: 18). In short, their capacity to engage with complex decision making is problematic.

Sen (1999) provides an alternative focus on the concept of human capability, being the ability of individuals to pursue livelihoods they value. Nevertheless, there is no coherent framework for determining the key factors which shape these livelihoods. The most substantial research effort has probably been on the notion of quality of life, and much of this dates from the 1980s and 1990s. The equilibrium modelling approach, which understands quality of life as being constituted of a balance between economic and amenity factors (Davies et al, 2001), is particularly relevant, although tending to be framed in the language of 'investment'. Nevertheless, the importance of particular factors remains indeterminate.

Climate and the environment have been recurrent quality of life concerns (Scott, 2010). More recent research has focussed on a range of general environmental influences (Henry et al, 2003) although climate continues to be an important consideration (Scott, 2010), especially in relation to life style migration (Benson and O'Reilly 2009). Social capital is another frequently discussed factor (Appleyard, 1992), focussing on how this increases flows of information from, and reduces the total costs of migration to, larger established migrant communities. Other factors include the importance of the quality of health care, as well as educational facilities. However, as emphasised above, there is no coherent theory which indicates which quality of life factors are most important. This varies across the life cycle and is contingent on the type of migration flow.

\section{Information overload and imperfect information}

Information (and knowledge) is critical to migration decision making. The idealised perfect knowledge assumptions of neo-classical theories are limiting (Stigler, 1961, Stiglitz, 2000) but there is little or no detailed analysis of how individuals deal with either imperfect and/or overload of information when making migration decisions. This will vary not only with the conditional access to information but also according to social and personality characteristics. Previous migration experience is one such characteristic - it represents a potential learning opportunity about migration in general, and specifically about what types of information are key to decision making (Baláž et al 2004).

Neo-classical economic theory has been a cornerstone of research on individual migration (for example, Sjaastad, 1962). Based on utility maximisation, it involves a number of simplifying assumptions including the following related to information, and information processing: (1) that individuals have all the available information for decision making; (2) that obtaining information involves no costs to the individual; (3) that individuals have unlimited processing capacity; (4) that migration attribute values and decision weights are known; and (5) that individuals use optimal analytical methods to make decisions. In reality decision making rarely reflects these idealised conditions. Instead, complex choices involve searching for large amounts of information, but this may be limited by: the costs of information acquisition (materially, or temporally) and the cognitive capacity of a decision-maker, that is bounded rationality (Simon, 1955). 
Moreover, individuals may only search for information they consider to be worthwhile, but this depends on attitudes, preferences and previous experience.

Neo-classical economic theory does refer to information, but usually only acknowledges the costs of information acquisition. Stigler (1961), for example, supposed that all relevant information can be obtained at a cost, and that at some point its marginal utility is less than its cost. This largely ignored imperfect information and Stiglitz (2000) emphasised that individuals screened information, selecting information based on particular criteria.

Missing information is clearly important in migration decision making, but is largely absent from the research literature. There is, however, an extensive generic literature that we can apply to migration. Four competing propositions are considered. First, that individuals substitute average values when faced with missing information (White and Koehler, 2004): a migrant lacking specific information about New York will focus on average USA data. Secondly, assuming that missing information is negative (Johnson and Levin, 1985); a migrant who knows wages but not housing costs in a destination, will assume the latter are relatively high. Thirdly, in contrast, positive information is assumed (Levin et al., 1985; Kivetz and Simonson, 2000); the migrant assumes there are no major health risks at a destination, if vaccinations are not required. Finally, assuming that individuals (migrants, in this instance) ignore missing information (Sanbonmatsu et al., 1992).

Garcia-Retamero and Rieskamp (2008) used experimental methods to test these propositions in a non-migration context, and found evidence that all four perspectives applied in different circumstances. Averages are substituted for missing information but only where randomly distributed. Negative/positive information is substituted when the missing information is conditionally distributed. Applying these ideas to migration, if income data is missing for a potential destination, migrants assume a hidden problem and substitute negative information. In contrast, if data on life satisfaction in a developed country is missing, positive information is substituted: it is assumed this is because life satisfaction tends to be high in all developed countries. Missing information tends to be ignored only when considered non-relevant. The four propositions may apply at different stages of the information search.

\section{Pre-defined versus constructed preferences}

Classical economics assume that all goals (material or non material) can be defined by one common denominator, utility, but psychology researchers assume that individuals simultaneously pursue multiple goals which are not necessarily complementary (Kranz and Kunreuther, 2007). Goals are not necessarily known/set in advance, but are constructed according to the specific decision-making context. Preferences play an important role in the search, selection and processing of information, but opinions differ as to whether these are predefined versus constructed (Warren et al, 2011). Some researchers contest whether preferences are always or even usually constructed, arguing that many decisions stem from stable, underlying preferences (Kivetz et al., 2008). 
The concepts of pre-defined versus constructed preferences can be applied to migration decisions as illustrated below:

(a) Underlying preferences: e.g. I always wanted to live in a Nordic country.

(b) Preferences constructed during the process: e.g. the more I considered which country to migrate to, the more likely it became I would decide on the Nordic countries.

(c) Constructing preferences anew in every choice: e.g. every time I looked at new information, my choice changed. I saw information on Norway and decided on Norway, but then I saw information on Denmark and changed my mind.

These different approaches point towards a different conceptualization of complex decision making which represents a significant extension to the neo-classical approach, and offers a more realistic perspective on complexity and information imperfections and overloads. The paper is concerned with how individuals engage in active information searches, which are informed by their preferences and by different (and changing) strategies for dealing with missing information. It is also concerned with how judgements are formed, and decisions made, under diverse and imperfect modes of access to information. Particular attention is given to differences between those with and without previous migration experience, as informing future migration intentions (Carlson 2013).

\section{Methodology}

The research was undertaken in Slovakia, which has one of the highest emigration rates in the EU27: 8\% the total labour force worked abroad in 2012 (SOSR, 2012) while 30,000 tertiary students (15\% of the total) studied abroad in 2011 (Eurostat, 2012), representing very high rates of brain drain and brain circulation (Baláž et al., 2004).

No time limits were imposed on the interviews and experiments which mostly lasted 2 3.5 hours. Despite the time consuming nature of the data collection, we obtained a relatively large purposive sample of 157 university students (advanced undergraduates and post-graduates in economics and psychology from the University of Economics and Comenius University in Bratislava, and the Agriculture University in Nitra), and recent graduates (including a small number of scientists, from the Slovak University of Technology). Students were accessed via their teachers, and recent graduates via the social networks of doctoral students at these universities: participants did not receive incentive payments.

The sample was balanced in terms of international migration experience and gender: 80 migrants (41 female and 39 male) and 77 non-migrants (38 female and 39 male). A participant was considered to have been a migrant if $s /$ he had spent at least six month studying or working abroad. Very few had stayed abroad for a period of several years. The average age was 25.0 for non-migrants (minimum 18, maximum 35), and 25.7 for migrants (minimum 20, maximum 33), an insignificant difference at the 0.05 level. $95 \%$ were students or graduates, while the other $5 \%$ were in professional or managerial occupations and had completed secondary education. 
Being a purposive sample, no claims are made about representativeness. However, the sample are all relatively young, and more than $80 \%$ have a social science or humanities background. This group constitutes 59.4\% of all graduates in Slovakia in 2011 (source: 2013 Institute of Information and Prognoses of Education: Statistical Yearbook of Higher Education. See www.uips.sk), and 54.4\% of all unemployed graduates in 2011. Their unemployment rate is therefore approximately average for all Slovak graduates, and they have a significant incentive to seek work abroad. A recent survey indicates that $65 \%$ of Slovak graduates were 'ready to seek a job in a neighbouring country and $53 \%$ in more distant countries in 2012: this is about 50\% higher than for the total population. (Profesia Newsletter 2013).

Various techniques are available for presenting, and analysing the use made of, information in experimental research (Reisen et al., 2008: 642) including Mouselab. This represents a computerized version of information boards (Payne et al., 1993), which provides data on information acquisition, such as which data cells are examined, in which order, and the time spent looking at each cell. Its main weakness is that participants can see the table of information, that is how it 'should' be structured. The Active Information Search records information less precisely than Mouselab, but the format of a dialogue, with an interviewer approximates to interrogating an informant (say, a trusted friend) in real life, while the participant does not see the structure of the information. It is, however, relatively time consuming, and has a lower response rate. Finally the Retrospective Verbal Protocol (RVP) represents a rich and detailed method of acquiring qualitative information, whereby the informant is interviewed after the decision making process. This is subject to the usual problems of post-rationalisation, fabricated memories, and imperfect memory. The main technique used in this research was Active Information Search, combined with Mouselab-style information search tracking and the addition of some verbal protocols. All the information was given verbally to the participant, rather than via software. The only exception was the final phase (complete information), where the information was provided on paper sheets to the participants. The interviewer recorded, all the individual's information searches, and reported these using the Mouselab method. We preferred working with paper copies, as computer screen ticking can involve insufficient reflection by the participants. The short verbal protocols involved statements in a few words about the major reasons for the choices made, that is immediate explanations at the end of each stage, rather than retrospectively. These are different to the time consuming RVP method which was not a realistic option given our already long experiments.

All participants were presented with the following hypothetical task:

'Please imagine, you graduated from University and found work with a multinational company which has branches around the world. You are offered an opportunity to obtain a good job and career advancement. Your employer, however, indicates that you would have to accept a company transfer to a foreign country for three years. You may choose from 10 countries. The job position (business representative) is the same in whichever country you choose. You are expected to learn the basics of the local language in one year. Extra pay will be received for working in 'demanding' countries'. 
Information was available on eight attributes for each country, including wages/costs and non-monetary costs and benefits. As discussed earlier, and reflecting the current state of the literature, any selection is necessarily arbitrary to some extent, but there is evidence to support those listed below. There were also practical limits to the number of variables that could be included, especially as the experiments already took 2-3 hours each. The eight variables selected were:

1. Monthly net wage (after tax), in Euros. These reflect actual wages (ILO, 2010), modified to include a 'risk premium' paid for working in some of the poorer, more 'difficult' countries.

2. Living costs in Euros based on minimum wages/living costs statistics (ILO, 2010).

3. Climate: the prevailing weather in their capital cities is classified as being cold, mild-cold, mild, Mediterranean, hot-dry, hot-humid.

4. The crime rate, based on homicide statistics (UNODC, 2011), which tend to be reported more consistently across crimes against property.*

5. Health, based on the number of vaccinations required (WHO, 2012).*

6. Language difficulty measured in terms of linguistic distance from Slovak, based on the Levenshtein distance concept (Serva and Petroni, 2008): 1 = Czech (very easy); 2 = other Slavonic (easy, e.g. Polish); $3=$ Germanic and Romanic (medium difficult, e.g. Danish), $4=$ other Indo-European (difficult, e.g. Farsi), 5 non-Indo-European (very difficult, e.g. Arabic); $6=$ non-Indo-European tonal (extremely difficult, e.g. Vietnamese).

7. Life satisfaction, based on the Satisfaction with Life Index (White, 2007).*

8. Personal freedom and security, based on the Civil Liberties Index (Freedom House, 2012$)^{*}$

$*$ = rescaled on scale of $1-6$

All the variables, except 1 and 2, are based on six point scales, and are presented in qualitative terms e.g. life satisfaction is high. It was made clear that these are average data for the destination countries rather than being specific to their migrant communities. When some participants asked about the meaning of the qualitative information (e.g. 'what does it mean that life satisfaction is high'), we explained the underlying statistics. However, such questions were rare, and no participants asked for information specific to migrants' experiences (e.g. of life satisfaction) in the destination countries.

Several other variables were considered but ultimately excluded due to pracrtical constraints, and specific reasons. Education data were not included as the sample is mostly constituted of young graduates, without children. Potential employment for partners was not included, in order to simplify the research. Religion was not directly included, but personal freedom is included. There was a lack of comparative data on anti-immigration feelings and social integration. Research on migrant networks indicates that social capital strongly influences the likelihood of out-migration (Massey et al., 1993, Palloni et al., 2001); this is simulated through including the countries with the highest numbers of Slovak migrants (the Czech Republic, UK and Ireland). No information is provided in the experiments about the size of migrant communities 
(Haug, 2008), although this is acknowledged to be important. This is because there are only a small number of countries with substantial numbers of Slovak migrants and it is assumed that this will be common existing knowledge. Providing such information makes it likely that participants could identify specific countries in the experiments where country name is withheld. The proximity of the destinations was not included because there is evidence that the proportions of students wishing to seek jobs in neighbouring countries $(65 \%)$ and more distant countries $(53 \%)$ were not very different (Profesia, 2013). Although housing opportunities are generally important in migration, this was not included in the study because it is probably of relatively less importance for young, single, migrants, than for those with families.

The design of the research tasks was adapted from Monti et al's (2009) study of complex financial decision making. In the three stage design, different tasks were presented under different access to information conditions. All three stages examine judgement formation, that is the decision weights attached to different information. The amount and type of the information used is recorded, as well as the order in which it is requested in the first two stages.

- Stage 1 is a simple binary choice experiment. Participants are presented with potential information on eight attributes for two unnamed countries but do not know how this is structured in the researcher's table. Limited access to information is simulated by only being allowed to request up to $50 \%$ of the items, necessitating assessment of their relevance. Countries are not named to avoid image influencing behaviour. The trial is repeated four times (a-d)

- Stage 2 is a complex choice experiment involving imperfect information. Individuals can search for as much information as they want on eight attributes for ten countries, that is between 0 and 80 units of information; the structure of the table is unseen. Countries are named, signalling surrogate information. At the end of the task, participants ranked the 10 potential destinations on a ten point scale. The participants also self-assess their knowledge, and their image, of each country on ten point scales. They combine previous knowledge with knowledge acquired during the experiments, permitting analysis of the extent to which preferences are constructed. The trial is repeated four times (1-4)

- Stage 3 is a complex choice with 'perfect' information experiment. Participants are presented with full information for 8 variables on 10 unnamed countries, clearly structured in a table. Countries are unnamed in order to isolate the image effect. Two sets of countries from Stage 2 (trials 2 and 3) and used in trials 5 and 6 in Stage 3 . The middle two countries from Stage 2 are selected as participants are more likely to remember data from the first and last experiments. We also changed the ordering of countries to minimize memory clues.

Each Stage consisted of a number of consecutive trials, with varying sets of countries, in order to examine the consistency of information search and judgement formation, while enabling observation of the effects of learning, constructing preferences, and forming/changing decision strategies. Differences between those with migrant and nonmigrant experiences are considered, but gender differences are not reported, partly because of space constraints and partly because there were relatively few significant differences. 


\section{Findings}

\section{Stage 1: information search and utilisation under different task conditions}

The binary choice task, repeated across four trials for two unnamed countries, simulated Stigler's (1961) proposition about the importance of information costs. Participants could obtain up to a maximum of one half of the total information available (4 out of 8 items for each country), assuming it would be too costly to consider the remainder.

The information most demanded (Table 1) was wages (accessed on $86.1 \%$ of occasions) and cost of living $(80.1 \%)$, consistent with the neo-classical emphasis on monetary factors. However, non-economic attributes, such as freedom, language and life satisfaction, were also important. Wages were the first information sought by the largest proportion of participants, followed by living costs. Other factors, such as climate, were rarely asked for first.

The Mann-Whitney U-test was applied to differences between migrants versus nonmigrants. Non migrants asked for more information on language difficulty (sig. 0.003), wages (sig. 0.048) and living costs (sig. 0.058) than migrants, while migrants sought more information on climate (sig. 0.024) and life satisfaction (sig. 0.018). That nonmigrants were more likely to ask about monetary attributes reflects uncertainty about the returns to migration. They were also more likely to ask about language difficulties, perhaps because migrants already had experience of coping with these. Analysis of the order in which particular attributes were requested (Table 2) indicates that wages were ranked first, and costs second, for both migrants and non-migrants. Migrants placed more emphasis on life satisfaction, health and climate in the first three trials.

\section{Stage 2: Complex choice with unlimited information}

In the second stage, the subject could request any information for items $1-8$ for 10 named countries, that is for between 0 and 80 items. This approximates to a complex choice, when large amounts of unordered information are potentially available. The choices are repeated in four trials with different sets of countries.

Neo-classical economic theory supposes that individuals use all available information when making decisions. In practice they have bounded rationality (Simon, 1955) and face potential information overload, understood as overabundance of incoming information in relation to the capacity of individual senses. Lee and Lee (2004), for example, found that the number of attributes, and the attribute level distribution (their scaling), are good predictors of how information overload influences consumer choice. Here, the average amount of information sought was 36.2 (45.3\%) of the 80 units). Only 13 participants $(8.3 \%)$ demanded all the available information, and most respondents tried to reduce the information overload.

The total information sought decreased modestly over the four trials $(36.2-32.5-30.6$ -29.8 units). This was statistically significant for Trials 1 versus 2 (Wilcoxon text, sig. 0.000), and Trials 2 versus 3 (sig. 0.017) but not for Trials 3 versus 4 (sig. 0.112). There 
was a learning effect across the trials, but it was relatively limited because individuals expressed what is really important to them in the first trial. In other words, their preferences in information search patterns, were far stronger than the learning effect. This experiment suggests they are both pre-set (Bettman et al,, 1998) and constructed (Warren et al,, 2011) preferences are fairly stable over the process of making choices about migration destinations.

Migrants asked for less information than non-migrants in all four trials, but the difference was insignificant at the 0.1 level (Figure 1). Non-migrants, however, displayed a more significant decrease in the information demanded between trials 1 and 2 (Wilcoxon test sig. 0.000) than migrants (sig. 0.015). There were similar differences between non migrants (sig, 0.035) and migrants (sig, 0.213) between trials 2 versus 3, but the decline was insignificant for both groups between trials 3 and 4. Migrants asked for less information at every point probably because their migration experiences facilitated the filtering of information. The decline in the amount of information demanded was greater for non-migrants, who had more to learn in this respect.

The analysis of which items of information had been requested (Table 1) indicates that the dominant attributes were wages $(86.5 \%)$ and living costs $(81.2 \%)$, followed by crime $(56.7 \%)$, health risks $(50.3 \%)$, level of freedom and democracy $(48.1 \%)$, life satisfaction $(45.1 \%)$, language $(28.2 \%)$ and climate $(15.3 \%)$. The attribute rankings were stable over the four trials (Table $1, \mathrm{~N}=157 \mathrm{x} 4=628$ ) and broadly similar to those observed in the binary choice experiment. The only significant difference was for language and this is understandable as the names of the countries were not revealed in the binary choice experiment, and these often indicated the difficulty of the language spoken. They mostly only asked about the languages spoken in less well known countries such as Paraguay or Oman.

The Mann-Whitney U-test indicated that non-migrants requested more information about wages and costs (sig. 0.014 and 0.002), and crime (sig. 0.042), but not about language. Revealing the name of the country may have removed an uncertainty which had been less troubling for migrants in the binary experiment. Migrants generally asked for less information than non-migrants, probably because they already had tacit knowledge of many aspects of migration. Previous migrants' greater concerns about life satisfaction are consistent with survey findings that foreign born populations have lower levels of life satisfaction than non migrants (Olgiati et al., 2012). Many studies of migrants and return migrants have also found similar differences in respect of life satisfaction and well being (De Jong et al., 2002: 857; Bălțătescu 2007: 79).

In summary, as expected higher decision weights were attached to those attributes with a higher degree of uncertainty (language difficulty, health risks, crime rate) in the binary than the complex choice experiments, because there was less information available and the countries were unnamed in the former. For example, relatively few individuals sought information about health risks in the UK or Denmark in Stage 2, probably substituting positive information for missing information. More participants asked for health information about countries they were less familiar with, but this was not universal, possibly indicating the substitution of negative information. Country image therefore seems to be a substitute for both positive and negative information. Most of 
the differences in information search patterns between those with and without migration experience also persisted in the complex unlimited choice experiment, with the main change from the binary experiment being the focus on language.

We now turn from judgements to decisions about specific destinations. Displaying the country names in Stage 2 meant their images were likely to have influenced the rankings. This is supported by the significant (0.000 level) correlation coefficients between country rankings, perceived knowledge of, and images of the countries. However, while the Spearman correlation coefficient for knowledge and ranking is medium low (0.379), that for image and ranking is medium high (0.602) as is the correlation for image and knowledge (0.605). Image, therefore, seems to be more closely associated than knowledge with the ranking of destination countries. In effect, the country names signal information to decision makers. However, image is informed by previous knowledge, and the highest correlation was between image and knowledge. Over the four trials, the correlations of both image and knowledge with country ranking increased, indicating learning. There were no significant differences between migrants and non-migrants.

In summary, all the participants learned how to manage potential information overload, by selectively seeking information. Migrants seemed somewhat more effective in their more frugal requests for information. However, the attribute rankings were similar over trials 1-4, and fairly similar in the complex choice and binary choice experiments, for migrants and non-migrants. The decision-making process - the country rankings - was significantly more associated with image than knowledge. There is some learning across trials, but this is relatively modest compared to the images held by individuals.

\section{Stage 3: Complex choice with complete information, but country names withheld}

Stage 3 assesses whether decisions (ranking of destinations) are affected by withholding information about the image of the country, while also providing complete information in a clearly structured format. In trials 5 and 6, the participants are presented with tables of the same ten countries used in Stage 2, trials 2 and 3, but now they are unnamed.

The main analytical focus is on eliciting decision weights, which are a function of attribute values and attribute weights. This research used three methods for eliciting decision weights under diverse experimental contexts, reflecting the different task conditions in Stage 2 and 3. First, we considered decision weights when making complex choices with unlimited information, when the country name is known. Most participants did not ask for all the available information, so decisions were mostly made under imperfect information conditions. It is difficult to compute 'objective' decision weights from imperfect decision matrices, but they can be derived from the participants' information search patterns - how frequently items were sought. The following decision weights were calculated: wages $21.0 \%$, living costs $19.7 \%$, crime rate $13.8 \%$, health risks $12.2 \%$, freedom and security $11.7 \%$, language $6.9 \%$ and climate $3.7 \%$. Secondly, we used short verbal protocols (obtained immediately after the task) to record the major reasons for the destination rankings. Verbal protocols about decision making are an important counterbalance to the first method because the frequency that information is requested may be more important for judgement formation than decision making. 
Thirdly, when making complex decisions with complete information for unnamed countries (trials 5 and 6, Stage 3), the decision making matrix was used to derive decision weights. Linear scale transformations converted all the $x_{i j}$ values to standardised $r_{i j}$ values on a $0-1$ scale:

$$
r_{i j}=\frac{x_{i j}-x_{j}{ }^{\min }}{x_{j}{ }^{\max }-x_{j}{ }^{\min }} \text { for the benefit, and } r_{i j}=\frac{x_{j}{ }^{\max }-x_{i j}}{x_{j}{ }^{\text {max }}-x_{j}{ }^{\text {min }}} \text { for the cost, criteria. }
$$

The transformation enabled the construction of decision matrices (systems of linear inequations) and computing decision weights based on transformed attribute values. The matrix values were independent variables while the country ranks, on a scale of 1-10, were dependent variables. We assumed linear relationships and used the simplex algorithm to compute decision weights. In about $10 \%$ of cases, linear solutions were unavailable, so decision weights were assumed to be non-linear and were determined by the Generalized Reduced Gradient (GRG) method.

Table 4 displays attribute weights elicited in the two diverse experimental conditions when country image is and is not available. The similarity of the weights is striking. Wages and living costs were the dominant attributes, and health risks and crime rates were important under both conditions. While attribute rankings were similar under both conditions, there were differences in the absolute values of the weights of selected attributes. Attribute values for 'wages' and 'living costs' are easier to estimate under imperfect information (trials 1-4) than attribute values for 'life satisfaction', which arguably are more abstract concepts and draw more on tacit knowledge. Therefore, information for some qualitative attributes was requested relatively more frequently, compared to wages and costs, under imperfect information conditions than under complete information conditions (trials 5-6, $\mathrm{N}=2 \times 157=314$ ). Nevertheless, the attribute importance rankings were similar in Stages 2 and 3.

There were limited differences (no significant Mann-Whitney U-test results) in decision weights by migration experience under complete information conditions and absence of country names (image) effect (Table 3 ). This suggests that information search patterns and judgement formation processes were 'invariable' across the observed migration groups.

Turning to comparisons of identical sets of countries under different information conditions in Stages 2 and 3, there were significant differences (Wilcoxon Signed Rank Test, sig. 0.05) in all the attribute weights between the two stages. Wages and living costs remained dominant attributes under complete information conditions (Stage 3), but their relative importance increased compared to unlimited information conditions (Stage 2). Although, some differences were observed between migrants and non migrants between Stages 2 and 3, there was more stability than change (see Table 3). Individuals were broadly consistent in their preferences when seeking information, indicating a strong underlying component in judgement formation.

As noted earlier, decision weights elicited from verbal protocols need not fully correspond to those elicited via the information frequency method, as they refer respectively to the decision making and judgement formation stages. However, they 
were mostly similar (Table 3) and the lower weights attached to costs in the decision weights elicited via the verbal protocols were the major difference in all three stages of the experiment. Arguably, the relatively high importance of living costs in the judgement formation stage is a manifestation of loss aversion (Tversky and Kahneman, 1992). Wage levels did broadly offset high living costs in some developed countries, and it can be proposed that the overall importance of living costs decreased accordingly in the decision-taking stage. This highlights the need to distinguish judgements and decisions.

Comparing identical sets of countries in trials 2 and 5, and trials 3 and 6, allows assessment of the influence of image. The average country rankings were broadly similar under both conditions (Table 4) but with some notable exceptions (significant at the 0.05 level for the total sample). The Czech Republic and the UK seemed less attractive, when the country name was displayed:, As these are major migration destinations for Slovaks, it suggests that the name of the country contributes to forming more realistic images. Finland, Taiwan and Oman seemed more attractive, when the country name was displayed: this may reflect relatively few Slovaks having real (tacit) knowledge of these countries. Iran seemed more unattractive, when its name was displayed, reflecting its weak image in Slovakia. Therefore, image seema to be an important factor.

Country image can be assumed to be strongly associated with particular attributes such as the degree of freedom and security. In contrast, other attributes may be more important when the country name is withheld, especially wages and living costs. Individual rankings produced under different experimental conditions were correlated to provide a measure of their similarity. The correlation coefficients for the ten countries ranged from $0.219-0.534$ in trials 2 versus 5: nine of the ten coefficients were significant (0.001 level). The correlation coefficients for trials 3 and 6 ranged from 0.229-0.584: eight of the ten were significant (0.001 level). These findings indicate there was some similarity in the attribute weights given by individuals under different experimental conditions, but that image is also an important predictor.

The participants generally first ranked those developed EU countries (Denmark, Norway, Finland, Sweden) which had the highest nominal wages and living costs, and were perceived as offering a good combination of earnings and quality of life. The inclusion of the UK, Ireland and the Czech Republic simulated the effects of migration networks. These destinations offered three advantages: language comfort, as most young Slovaks spoke English, while the Czech and Slovak languages are very similar; favourable wage-living cost ratios; and opportunities to rely on extensive networks of Slovak migrants. The UK, Ireland and the Czech Republic were usually ranked in second or third places. Taiwan and Japan had similar rankings: they offered high nominal wages, but low language comfort and limited Slovak migrant networks. The lowest rankings were associated either with less developed countries in Asia which had weak images (Vietnam, Laos, Cambodia, Pakistan) or oil producing countries that the participants had little knowledge of (Qatar, Oman). In the latter, their lack of image probably outweighed the potential economic gains from migration. 
There were some differences in the country rankings between those with and without migration experience (Table 4). Migrants assigned higher rankings than non migrants to established migration destinations (the UK, Czech Republic and Ireland). Non-migrants had less negative opinions of migration to distant, and/or less developed, 'exotic' destinations (Laos, Guatemala, Chile, Argentina), perhaps because they had less tacit knowledge of migration realities. There were more differences between migrants and non-migrants when country names were displayed, which again indicates the influence of country image on country ranking.

In summary, Stage 3 indicated that attribute rankings were similar under unlimited but imperfect information, and complete information conditions. Some attributes, however, were given lower weights under complete information, especially the more abstract noneconomic attributes. This suggests that some of the more abstract features, such as security and freedom, seem more important when individuals have no information about them. Once some information is obtained, they may seem less formidable barriers. This change was reported for both migrants and non migrants, but was more pronounced under complete information conditions (Stage 3). It partly explains, why the country rankings are more similar, but not identical, for migrants and non-migrants in the complete information condition compared to the complex and unlimited choice condition. Moreover, the similar, but not identical, patterns of information search and analytical methods used by the migrants and non migrants may result in different decisions about migration destinations. Former migrants were more likely than non migrants to prefer established Slovak migration destinations (UK, Ireland, Czech Republic). Non-migrants, on the other hand, had less unfavourable opinions about more unusual and exotic destinations, reflecting their lack of experience of migration.

\section{Conclusions}

This research has sought to make an original contribution to migration research, though providing - as far as we know - the first systematic application of complex choice concepts and methods, and one of the few applications of any experimental methods, to migration, especially to non-economic factors (McKenzie and Yang, 2010). It responds to the limitations of neo-classical approaches (Stigler, 1961, Stiglitz, 2000). We investigated the following issues:

Which attributes (economic and non-economic) are important when choosing migration destinations, and how much weight is attached to them? Our findings provide an extension of the neo-classical focus on monetary outcomes, by providing measures of psychic costs and returns (Sjaastad, 1962: 84). Although wages and costs are the dominant attributes, their combined share in decision weights ranged from only $28.2 \%$ to $49.0 \%$. The remainder was explained by non-economic factors such as health, crime, and personal freedom and security. Three different methods for eliciting decision weights for particular migration attributes produced remarkably consistent rankings, underlining the importance of the non-monetary costs and benefits of migration.

How judgements on migration destinations are formed? The choice of migration destination is partially dependent on (a) incumbent preferences revealed, and (b) preferences constructed, during decision-making. Consistent rankings of migration 
attributes and destination countries across repeat trials, with named and unnamed countries, and different information conditions, indicate relatively strong stable underlying preferences in migration choice. This is in line with Kivetz, et al. (2008), rather than Bettman et al's (1998) contention that choice is always constructed. Incumbent preferences seem to arise from images which signal information (Stiglitz, 2000) and existing knowledge of migration destinations.

How potential migrants cope with information overload and imperfect information, and which information is considered relevant for migration decisions? The participants faced information overload in Stages 2 and 3. Very few participants used all the available information in the Stage 2 experiment with unlimited information, and on average accessed less than one half. This approximates the real world of imperfect information (Stiglitz, 2000) that migrants encounter. There was also a modest learning effect, as the amount of information sought decreased across four successive trials. Image and existing knowledge help reduce information overload, through surrogating some information. Both positive (Kivetz and Simonson, 2000) and negative surrogating (Johnson and Levin 1985) occur in migration decision making under experimental conditions: for example, information about crime in Japan is not requested because it is a highly developed country, but is requested for some less developed countries. This is consistent with Garcia-Retamero and Rieskamp's (2008) findings about surrogating in a different context.

Is previous migration experience important in choosing migration destinations? There were some differences in the frequency attributes were requested by those with and without migration experience, although varying to some extent across different experimental conditions. Migrants were more concerned with life satisfaction and climate, while non-migrants focussed more on language difficulty and wages. Migrants already possess some tacit knowledge about migration and deal with information overload more efficiently than non-migrants: they seek less information as they probably know which information is important to them. Some interesting questions arise. Our research, for example, confirmed that migrants were more concerned with life satisfaction than non-migrants (as indicated by Olgiati et al., 2012, De Jong et al., 2002, and Bălțătescu, 2007). Further experimental research could compare life satisfaction before and after migration and help establish the arrow of causality

This paper has explored one application of experimental methods in the analysis of migrant decision making but suggests that there is considerable potential to extend their utilisation. They can be used to provide information that is not obtainable with other methods, such as eliciting decision weights (monetary and non-monetary in particular). Moreover, they simulate the process of decision making, and avoid the postrationalisation and imperfect memory recall which characterises most questionnaire and interview-based data. One of the key advantages is the insights they provide into cause and effect relationships, via the manipulation of particular variables: this is widely considered to be the research approach that allows the most precise control to be exercised over the influence of particular variables, such as complete versus incomplete information. They can also be replicated (Hole 2012; Kagel and Roth 1995). 
Of course there are also disadvantages in using experimental methods (Samuelson 2005), including the time consuming nature of data collection. The interviews/experiments in this paper were mostly of 2-3.5 hours duration. Other limitations include the impossibility of replicating real world decision making in the artificial setting of a laboratory, where the implications of decision making are not realised. Moreover, only a narrow range of behaviour is usually analysed: for example, the data presentation involves a degree of selectivity and structuring of data provision (only eight variables were included in our research design) and searching which only partly reflects the complexity of actual individual decision making.

It is also virtually impossible to analyse representative samples using experimental methods, so that generalization is not constrained. This research was performed on a sample of Slovak students and graduates, and their behaviour, decision making and judgements reflect their age and education, as well as the economic, social and cultural environment in Slovakia in the early 2010s. The Slovak participants, for example, had more information about, and cultural attachments to, European than Asian countries. Additionally, Slovakia was a middle-high income country, and most of the migrants are driven by the attraction of higher wages rather than escaping from extreme poverty. Migrants from low-income countries would probably associate higher decision weights with the economic attributes of migration. Although no claim can be made that the sample was representative, the age structure (young adults) was typical for migrants from Slovakia, and for company channelled professional/managerial international mobility. There is therefore a need for further research to consider a wider range of migration types, including extra-company mobility, non-labour migration, refugees, migrants from different cultural backgrounds, and family decision-making processes.

In conclusion, there is a need for further research both to evaluate the contribution of experimental research methods to understanding the complexity of migration decision making, and to validate the findings of this study with respect to search procedures under imperfect information conditions, the role of image versus knowledge, learning processes, and decision weights. Beyond this, we contend that there is considerable scope for a wider application of experimental methods in migration research.

\section{References}

Appleyard R. 1989. Migration and development: Myths and reality. International Migration Review 23: 486-99

Bălțătescu S. 2007. Central and Eastern Europeans migrants' subjective quality of life. A comparative study. Journal of Identity and Migration Studies. 1 : 67-81.

Baláž V, Williams AM and Kollár D. 2004. Temporary versus permanent youth brain drain: economic implications. International Migration 42: 3-34.

Benson M and O'Reilly K. 2009. Migration and the search for a better way of life: a critical exploration of lifestyle migration. The Sociological Review 57: 608-625.

Bettman JR, Luce MF and Payne JW. 1998: Constructive consumer choice processes, Journal of Consumer Research. 25: 187- 217.

Borjas G.J. 1989. Economic theory and international migration. International Migration, Review 23: 457-85 
Carlson S. 2013. Becoming a mobile student - a processual perspective on German degree student mobility, Population Space and Place 19: 168-180

Central Office of Labour, Social Affairs and Family of the Slovak Republic 2013. Unemployment of Graduates Statistics, available at:

http://www.upsvar.sk/statistiky/nezamestnanost-absolventistatistiky.html?page_id=1252

Davies P, Greenwood M, Li H. 2001. A conditional logit approach to US state-to-state migration. Journal of Regional Science 41: 337-360.

De Jong GF. 1999: Choice processes in migration behavior, chapter 13, 273-292. In Migration and Restructuring in the United States, Pandit K, Withers SD. (eds): Rowman and Littlefield Publishers, New York: Inc.

De Jong GF, Chamratrithirong A. and Tran QG. 2002. For better, for worse: Life satisfaction consequences of migration. International Migration Review 36(3) : 838863.

Erasmus AC, Boshoff E and Rousseau GG. 2001. Consumer decision-making models within the discipline of consumer science: a critical approach. Journal of Family Ecology and Consumer Sciences 29: 82-90.

Eurostat 2012. Student mobility and foreign students in tertiary education. Available at: http://epp.eurostat.ec.europa.eu/portal/page/portal/statistics/search_database [accessed 18 April 2013].

Fisher K E. 2004. Information behaviour of migrant Hispanic farm workers and their families in the Pacific Northwest" Information Research, 10 paper 199 [Available at http://InformationR.net/ir/10-1/paper199.html]

Freedom House 2012. Freedom in the World 2012. Available at http://www.freedomhouse.org/report/freedom-world/freedom-world-2012 [accessed 18 April 2013].

García-Retamero R and Rieskamp J. 2008: Adaptive mechanisms for treating missing information: A simulation study. The Psychological Record 58: 547-568.

de Haas H. 2007. Migration and Development: a Theoretical Perspective. Bielefeld: Center on Migration, Citizenship and Development; Working Paper 29.

de Haas H. 2010. Migration and development: a theoretical perspective. International Migraiton Review 44: 227-264

Halfacree K. 2004. A utopian imagination in migration's terra incognita?

acknowledging the non-economic worlds of migration decision-making. Population

Space Place, 10: 239-253.

Haug S. 2008. Migration networks and migration decision-making. Journal of Ethnic and Migration Decision-Making 34: 585-605

Helliwell J, Layard R. and Sachs J. 2012. World Happiness Report, The Earth Institute Columbia University.

Henry S, Boyle P, Lambin E F. 2003. Modelling inter-provincial migration in Burkina Faso, West Africa: the role of socio-demographic and environmental factors, Applied Geography, 23: 115-136

Hole G. 2012. Experimental design. In Research Methods in Psychology, Breakwell, G M, Smith J A and Wright D B. London: Sage.

ILO, International Labour Office. 2010. Global Wage Report 2010/2011 Wage policies in times of crisis. Geneva: International Labour Office. 
Johnson RD and Levin IP. 1985. More than meets the eye: The effect of missing information on purchase evaluations. Journal of Consumer Research 12: 169-177.

Kivetz R and Simonson I. 2000. The effects of incomplete information on consumer choice. Journal of Marketing Research 37: 427-448.

Kivetz R, Netzer O, Schrift R. 2008. The synthesis of preference: bridging behavioral decision research and marketing science. Journal of Consumer Psychology 18: 179186.

Krantz DH and Kunreuther HC. 2007. Goals and plans in decision making. Judgment and Decision Making 2: 137-168.

Lee BK and Lee WN. 2004. The effect of information overload on consumer choice quality in an on-line environment, Psychology and Marketing 21: 159-183.

Levin IP, Johnson RD, Ruso CP and Deldin PJ. 1985. Framing effects in judgment tasks with varying amounts of information. Organizational Behavior and Human Decision Processes 36(3): 362-377.

MacKenzie D and Yang D. 2010. Experimental Approaches in Migration Studies The World Bank Development Research Group, Finance and Private Sector Development Team. Policy Research Working Paper No 5395.

Massey DS, Arango J, Hugo G, Kouaouc, A, Pellegrino A and Taylor JE. 1993. Theories of international migration: A review and appraisal. Population and Development Review 19: 431-466.

Monti M, Martignon L, Gigerenzer G and Berg N. 2009. The impact of simplicity on financial decision-making, Proceedings of the 31st Annual Conference of the Cognitive Science Society 386 : 1846-1851.

Olgiati A, Calvo R and Berkman L. 2012. Are migrants going up a blind alley? Economic migration and life satisfaction around the world: Cross-national evidence from Europe, North America and Australia. Social Indicators Research, DOI 10.1007/s11205-012-0151-4.

Palloni A, Massey DS, Ceballos M, Espinosa K and Spittel M. 2001. Social capital and international migration: A test using information on family networks. American Journal of Sociology 106: 1262-1298.

Payne JW, Bettman JR and Johnson EJ. 1993. The Adaptive Decision Maker. New York: Cambridge University Press.

Profesia Newsletter 2013. V susednom štáte je ochotných pracovat' 43\% l'udí zo Slovenska [Some $43 \%$ Slovaks are ready to seek for job in a neighbouring country]. Profesia Newsletter, April 2013,

http://www.profesia.sk/cms/newsletter/april-2013/v-susednom-state-je-ochotnychpracovat-43-ludi-zo-slovenska/44254

Reisen N, Hoffrafe U and Mast FW. 2008. Identifying decision strategies in a consumer choice situation. Judgment and Decision Making 3: 641-658.

Samuelson L. 2005. Economic theory and experimental economics, Journal of Economic Literature, 43: 65-107.

Sanbonmatsu DM, Kardes FR and Herr PM. 1992. The role of prior knowledge and missing information in multiattribute evaluation. Organizational Behavior \& Human Decision Processes 51: 76-91. 
Scott A J. 2010. Jobs or amenities? Destination choices of migrant engineers in the USA. Papers in Regional Science 89: 43-63.

Sen A. 1999. Development as Freedom. New York: Anchor Books

Serva M and Petroni P. 2008. Indo-European languages tree by Levenshtein distance. A Letters Journal Exploring the Frontiers of Physics 81: 68005.

Simon HA. 1955. A behavioral model of rational choice. The Quarterly Journal of Economics 69: 99-118.

Sjaastad LA. 1962. The costs and returns of human migration. The Journal of Political Economy 70: 80-93.

Skeldon R. 1997. Migration and Development: A Global Perspective. Harlow: Longman

Solomon M, Bamossy G, Askegaard S, and Hogg MK. 2006. Consumer Behaviour. European Perspective. Third Edition. Prentice Hall.

SOSR, Statistical Office of the Slovak Republic. 2012. Labour Force Survey. Available at: http://portal.statistics.sk/showdoc.do?docid=23558 [accessed 18 April 2013].

Stigler G. 1961. Economics of Information. The Journal of Political Economy 69: 213225.

Stiglitz JE. 2000. The contribution of the economics of information to twentieth century economics. The Quarterly Journal of Economics 115: 1441-1478.

Tversky A and Kahneman D. 1992. Advances in prospect theory: Cumulative representation of uncertainty. Journal of Risk and Uncertainty 5: 297-323.

UNODC, United Nations Office on Drugs and Crime (2011). Global Study on

Homicide. Trends, Context, Data. Vienna: UNODC. Available at: http://www.unodc.org/documents/data-and-

analysis/statistics/Homicide/Globa_study_on_homicide_2011_web.pdf [accessed 18 April 2013].

Warren C, McGraw AP and van Boven L. 2011. Values and preferences: defining preference construction. Wiley Interdisciplinary Reviews: Cognitive Science 2: 193-205.

White CM and Koehler DJ. 2004. Missing information in multiple-cue probability learning. Memory \& Cognition 32: 1007-1018.

White A. 2007. A global projection of subjective well-being: A challenge to positive psychology? Psychtalk 56: 17-20.

WHO, World Health Organisation. 2012. International Travel and Health 2012 Edition, Geneva: World Health Organisation. 
Table 1: Percentage of accessed information for particular migration attributes in binary and complex choices

\begin{tabular}{|c|c|c|c|c|c|c|c|c|}
\hline Mode of choice & \multicolumn{8}{|c|}{ Health risks language Freedom \& security Wage in EUR Crime rate Life satisfaction climate Living costs. EUR } \\
\hline & \multicolumn{8}{|c|}{ Binary choice, limited access to information, no display of country names } \\
\hline trial a & 49.7 & 52.2 & 38.9 & 86.0 & 40.1 & 32.5 & 26.1 & 87.9 \\
\hline trial b & 51.0 & 45.2 & 45.9 & 84.7 & 46.5 & 36.3 & 28.7 & 77.1 \\
\hline trial c & 41.4 & 42.7 & 43.3 & 87.3 & 46.5 & 45.2 & 29.9 & 79.0 \\
\hline trial d & 44.6 & 45.2 & 50.3 & 86.6 & 42.7 & 42.7 & 29.9 & 76.4 \\
\hline \multirow[t]{2}{*}{ average } & 46.7 & 46.3 & 44.6 & 86.1 & 43.9 & 39.2 & 28.7 & 80.1 \\
\hline & \multicolumn{8}{|c|}{ Complex choice, unlimited access to information, country names displayed } \\
\hline trial 1 & 55.4 & 33.8 & 53.5 & 93.6 & 59.9 & 48.4 & 17.8 & 88.5 \\
\hline trial 2 & 51.6 & 28.0 & 47.8 & 89.8 & 58.0 & 43.3 & 15.3 & 83.4 \\
\hline trial 3 & 45.9 & 26.8 & 46.5 & 83.4 & 54.1 & 44.6 & 14.0 & 78.3 \\
\hline trial 4 & 48.4 & 24.2 & 44.6 & 79.0 & 54.8 & 43.9 & 14.0 & 74.5 \\
\hline Average & 50.3 & 28.2 & 48.1 & 86.5 & 56.7 & 45.1 & 15.3 & 81.2 \\
\hline
\end{tabular}

$\mathrm{N}=157$ participants 
Table 2: Ranking of importance of migration factors in binary choice (\% distribution across all 8 rankings for each item of information)

\begin{tabular}{|c|c|c|c|c|c|c|c|c|c|}
\hline Non- & 1 question & 2 questions & 3 questions & 4 questions & 5 questions & 6 questions & 7 questions & Q1-Q3 & Total \\
\hline wage & 51.3 & 22.7 & 15.2 & 9.4 & 1.4 & 0.0 & 0.0 & 89.2 & 100.0 \\
\hline language & 32.5 & 16.0 & 25.8 & 19.6 & 3.7 & 1.8 & 0.6 & 74.2 & 100.0 \\
\hline health & 30.3 & 14.8 & 15.5 & 23.2 & 12.0 & 3.5 & 0.7 & 60.6 & 100.0 \\
\hline climate & 14.5 & 21.1 & 22.4 & 31.6 & 1.3 & 7.9 & 1.3 & 57.9 & 100.0 \\
\hline life & 14.0 & 16.8 & 20.6 & 38.3 & 6.5 & 0.9 & 2.8 & 51.4 & 100.0 \\
\hline freedom & 13.7 & 21.4 & 16.8 & 37.4 & 6.9 & 3.1 & 0.8 & 51.9 & 100.0 \\
\hline crime & 11.1 & 14.3 & 39.7 & 24.6 & 8.7 & 0.8 & 0.8 & 65.1 & 100.0 \\
\hline Costs of life & 3.5 & 50.2 & 29.6 & 12.8 & 2.3 & 1.2 & 0.4 & 83.3 & 100.0 \\
\hline Migrants & 1 question & 2 questions & 3 questions & 4 questions & 5 questions & 6 questions & 7 questions & Q1-Q3 & Total \\
\hline wage & 50.8 & 28.0 & 12.1 & 6.8 & 1.5 & 0.8 & 0.0 & 90.9 & 100.0 \\
\hline language & 19.5 & 14.1 & 27.3 & 31.3 & 6.3 & 1.6 & 0.0 & 60.9 & 100.0 \\
\hline health & 36.4 & 17.9 & 21.2 & 18.5 & 2.6 & 2.6 & 0.7 & 75.5 & 100.0 \\
\hline climate & 21.2 & 9.6 & 32.7 & 26.0 & 7.7 & 2.9 & 0.0 & 63.5 & 100.0 \\
\hline life & 16.5 & 20.9 & 22.3 & 32.4 & 5.0 & 2.2 & 0.7 & 59.7 & 100.0 \\
\hline freedom & 20.1 & 16.8 & 24.2 & 30.2 & 6.0 & 2.7 & 0.0 & 61.1 & 100.0 \\
\hline crime & 10.0 & 16.0 & 31.3 & 37.3 & 4.7 & 0.7 & 0.0 & 57.3 & 100.0 \\
\hline Costs of life & 7.5 & 45.2 & 27.4 & 16.6 & 2.5 & 0.8 & 0.0 & 80.1 & 100.0 \\
\hline
\end{tabular}

Notes: No participant requested eight pieces of information for any single country, so the maximum number of attributes that were searched for was seven

$\mathrm{N}=157$

Figures over $20 \%$ shown in bold 
Table 3: Average country rankings; total and migration differences

\begin{tabular}{|c|c|c|c|c|c|c|c|c|c|c|}
\hline \multirow[b]{2}{*}{ Trial 1} & \multicolumn{10}{|c|}{$\begin{array}{l}\text { COUNTRY NAMES DISPLAYED } \\
\end{array}$} \\
\hline & Norway & Slovenia & Britain & Czech & Brazil & Hong Kong & Peru & Malaysia & Philippines & Qatar \\
\hline Total & 4.01 & 4.03 & 4.16 & 5.26 & 5.43 & 5.43 & 6.25 & 6.61 & 6.74 & 7.09 \\
\hline Non-migrant & 3.69 & 3.95 & 3.88 & 5.35 & 5.82 & 5.55 & 6.57 & 6.79 & 6.60 & 6.81 \\
\hline Migrant & 4.31 & 4.10 & 4.43 & 5.18 & 5.05 & 5.33 & 5.94 & 6.44 & 6.88 & 7.36 \\
\hline Trial $2(=5)$ & Finland & Britain & Czech & Argentina & Taiwan & Poland & Honduras & Vietnam & Oman & Iran \\
\hline Total & 2.73 & 3.50 & 4.33 & 5.00 & 5.06 & 5.11 & 6.34 & 6.99 & 7.14 & 8.78 \\
\hline Non-migrant & $3.08 *$ & 4.17 & 5.08 & 4.51 & $4.97 *$ & 5.29 & 5.77 & 6.47 & 7.09 & 8.58 \\
\hline Migrant & $2.40^{*}$ & 2.86 & 3.61 & 5.48 & $5.15^{*}$ & 4.95 & 6.90 & 7.49 & 7.19 & 8.98 \\
\hline Trial 3 ( = 6) & Denmark & Ireland & Japan & Czech & Croatia & Chile & Guatemala & Turkey & Kuwait & Pakistan \\
\hline Total & 2.47 & 3.67 & 3.85 & 4.76 & 4.87 & 5.42 & 6.75 & 7.02 & 7.13 & 9.06 \\
\hline Non-migrant & 2.68 & $4.25^{*}$ & 3.57 & $5.36^{*}$ & 5.09 & $4.88^{*}$ & $6.23^{*}$ & 6.95 & 7.06 & 8.92 \\
\hline Migrant & 2.28 & $3.11 *$ & 4.11 & $4.19 *$ & 4.66 & $5.94 *$ & $7.25 *$ & 7.09 & 7.19 & 9.19 \\
\hline Trial 4 & Sweden & Ireland & Czech & Korea & Mexico & Serbia & Paraguay & Bahrain & Laos & Kazakhstan \\
\hline Total & 2.10 & 3.38 & 4.25 & 5.32 & 5.38 & 5.45 & 6.17 & 6.93 & 7.59 & 8.43 \\
\hline Non-migrant & 2.27 & $4.05^{*}$ & $5.01 *$ & 5.16 & 5.16 & 5.77 & $5.42 *$ & 6.87 & $7.10^{*}$ & 8.19 \\
\hline \multirow[t]{2}{*}{ Migrant } & 1.94 & $2.73 *$ & $3.51 *$ & 5.49 & 5.60 & 5.15 & $6.90 *$ & 6.99 & $8.05^{*}$ & 8.65 \\
\hline & \multicolumn{10}{|c|}{ COUNTRY NAMES WITHHELD } \\
\hline Trial 5 ( = 2) & Finland & Britain & Taiwan & Czech & Poland & Argentina & Oman & Honduras & Vietnam & Iran \\
\hline Total & 3.41 & 3.35 & 4.02 & 4.69 & 5.24 & 5.97 & 6.11 & 7.01 & 7.39 & 7.81 \\
\hline Non-migrant & 3.47 & 3.66 & $3.40^{*}$ & $5.51^{*}$ & 5.64 & $5.38^{*}$ & 5.88 & 6.71 & 7.31 & 8.04 \\
\hline Migrant & 3.36 & 3.05 & $4.61^{*}$ & $3.90^{*}$ & 4.86 & $6.55^{*}$ & 6.33 & 7.29 & 7.46 & 7.59 \\
\hline Trial $6(=3)$ & Denmark & Ireland & Japan & Czech & Chile & Croatia & Guatemala & Kuwait & Turkey & Pakistan \\
\hline Total & 2.22 & 3.75 & 4.57 & 4.77 & 5.06 & 5.53 & 6.90 & 6.25 & 7.49 & 8.46 \\
\hline Non-migrant & 2.19 & 3.75 & 4.30 & 5.14 & 4.77 & 5.79 & 6.61 & 6.21 & 7.45 & 8.78 \\
\hline Migrant & 2.25 & 3.75 & 4.83 & 4.41 & 5.34 & 5.28 & 7.19 & 6.29 & 7.53 & 8.15 \\
\hline
\end{tabular}

Notes: a) $*=$ significant on the 0.05 level, also shown in bold, Mann-Whitney U-test b) Ranked form 1 (highest) to 10 (lowest); c) $\mathrm{N}=157$. . 
Table 4: Attribute weightings under different experimental conditions and methods of computation (\%)

\begin{tabular}{|c|c|c|c|c|c|c|c|c|c|}
\hline Experimental conditions & Health risks & Language & Freedom \& security & Wage in EUR & Crime rate & Life satisfaction & Climate & Living costs. EUR & Total \\
\hline \multicolumn{10}{|c|}{ Stage 1: Binary choice, with limited information (trials a-d) } \\
\hline Total & 11.2 & 11.2 & 10.7 & 20.7 & 10.6 & 9.4 & 6.9 & 19.3 & 100.0 \\
\hline Migrants & 11.5 & 9.6 & 11.3 & 19.8 & 11.5 & 10.4 & 7.6 & 18.4 & 100.0 \\
\hline Non-migrants & 11.1 & 12.7 & 10.2 & 21.6 & 9.9 & 8.4 & 6.0 & 20.1 & 100.0 \\
\hline Binary choice - verbal protocols & 11.0 & 8.3 & 10.9 & 26.0 & 14.4 & 10.7 & 5.2 & 13.6 & 100.0 \\
\hline Non-migrants & 10.2 & 10.1 & 10.6 & 27.2 & 13.8 & 10.4 & 4.3 & 13.4 & 100.0 \\
\hline \multicolumn{10}{|c|}{ Stage 2: Complex choice with unlimited information (trials 1-4) } \\
\hline Total & 12.2 & 6.9 & 11.7 & 21.0 & 13.8 & $\mathbf{1 1 . 0}$ & 3.7 & 19.7 & 100.0 \\
\hline Migrants & 12.6 & 6.5 & 12.4 & 20.9 & 13.2 & 11.1 & 3.9 & 19.4 & 100.0 \\
\hline Non-migrants & 12.1 & 7.1 & 11.0 & 21.1 & 14.4 & 10.9 & 3.5 & 20.0 & 100.0 \\
\hline Complex choice - verbal protocols & 9.9 & 7.0 & 13.0 & 23.8 & 15.5 & 10.0 & 5.0 & 15.8 & 100.0 \\
\hline Migrants & 8.7 & 8.2 & 13.0 & 23.6 & 15.3 & 11.1 & 5.1 & 15.0 & 100.0 \\
\hline Non-migrants & 11.1 & 5.8 & 13.0 & 23.9 & 15.8 & 9.0 & 4.8 & 16.6 & 100.0 \\
\hline Total & 9.9 & 5.5 & 8.5 & 25.8 & 14.5 & 6.0 & 6.9 & 23.2 & 100.0 \\
\hline Migrants & 9.5 & 5.6 & 8.7 & 25.8 & 13.7 & 5.9 & 6.9 & 23.9 & 100.0 \\
\hline Non-migrants & 10.2 & 5.4 & 8.2 & 25.7 & 15.2 & 5.9 & 6.9 & 22.4 & 100.0 \\
\hline Complete information - verbal protocol & 15.1 & 8.6 & 14.9 & 19.2 & 15.7 & 10.2 & 7.2 & 9.0 & 100.0 \\
\hline Migrants & 13.6 & 9.3 & 15.4 & 18.5 & 15.4 & 11.7 & 8.6 & 7.5 & 100.0 \\
\hline Non-migrants & 16.9 & 7.8 & 14.2 & 20.1 & 16.1 & 8.6 & 5.6 & 10.7 & 100.0 \\
\hline
\end{tabular}

$\mathrm{N}=157$ 